\title{
ON THE STABILITY OF A GENERALIZED CUBIC FUNCTIONAL EQUATION
}

\author{
Heejeong Koh and DongSeung Kang
}

ABSTRACT. In this paper, we obtain the general solution of a generalized cubic functional equation, the Hyers-Ulam-Rassias stability, and the stability by using the alternative fixed point for a generalized cubic functional equation

$$
\begin{aligned}
& 4 f\left(\sum_{j=1}^{n-1} x_{j}+m x_{n}\right)+4 f\left(\sum_{j=1}^{n-1} x_{j}-m x_{n}\right)+m^{2} \sum_{j=1}^{n-1} f\left(2 x_{j}\right) \\
= & 8 f\left(\sum_{j=1}^{n-1} x_{j}\right)+4 m^{2} \sum_{j=1}^{n-1}\left(f\left(x_{j}+x_{n}\right)+f\left(x_{j}-x_{n}\right)\right)
\end{aligned}
$$

for a positive integer $m \geq 1$

\section{Introduction}

The stability theory of functional equations started with the talk of S. M. Ulam held at the Wisconsin University in 1940 as follows: Under what condition does there exist an additive mapping near an approximately additive mapping? see [19].

The first partial solution to Ulam's question was provided by D. H. Hyers [7]. Let $X$ and $Y$ are Banach spaces with norms $\|\cdot\|$ and $\|\cdot\|$, respectively. Hyers showed that if a function $f: X \rightarrow Y$ satisfies the following inequality

$$
\|f(x+y)-f(x)-f(y)\| \leq \epsilon
$$

for all $\epsilon \geq 0$ and for all $x, y \in X$, then the limit

$$
a(x)=\lim _{n \rightarrow \infty} 2^{-n} f\left(2^{n} x\right)
$$

exists for each $x \in X$ and $a: X \rightarrow Y$ is the unique additive function such that

$$
\|f(x)-a(x)\| \leq \epsilon
$$

for any $x \in X$.

Thirty seven years after Hyers's Theorem, Th. M. Rassias in his paper [12], provided a remarkable generalization of Hyers's result by allowing for the first

Received March 8, 2008.

2000 Mathematics Subject Classification. 39B52.

Key words and phrases. Hyers-Ulam-Rassias stability, cubic mapping. 
time in the subject of functional equations and inequalities the Cauchy difference to be unbounded. This fact rekindled interest of several mathematicians worldwide in the study of several important functional equations of several variables. In 1990, Th. M. Rassias [16] during the 27th International Symposium on Functional Equations asked the question whether his Theorem can also be proved for all real values of $p$ that are greater or equal to one. In 1991, Z. Gajda following Th. M. Rassias' approach to stability of functional equations obtained an affirmative solution to this question for all real values of $p$ that are strictly greater than one; see [5].

The cubic function $f(x)=c x^{3}(c \in \mathbb{R})$ satisfies the functional equation

$$
f(2 x+y)+f(2 x-y)=2 f(x+y)+2 f(x-y)+12 f(x) .
$$

The equation (1.1) was solved by Jun and Kim [11]. They proved that a function $f: X \rightarrow Y$ is a solution of the equation (1.1) if and only if there exists a function $F: X \times X \times X \rightarrow Y$ such that $f(x)=F(x, x, x)$ for all $x \in X$, and $F$ is symmetric for each fixed one variable and is additive for fixed two variables; see [11]. We promise that by a cubic function we mean every solution of the equation (1.1) is called a cubic function. Also, the equation (1.1) is equivalent to the following equation (see [2, Lemma 2.1]);

$$
f(x+2 y)+f(x-2 y)+f(2 x)=2 f(x)+4 f(x+y)+4 f(x-y) .
$$

Recently, the $n$-dimensional cubic functional equation was investigated by Chu and Kang [2]. Also, G. Isac and Th. M. Rassias [10] were the first mathematicians to apply the Hyers-Ulam-Rassias stability approach for the proof of new fixed point theorems. For an extensive account on the development of fixed point theory and related topics the reader is referred to the book; see [8].

In this paper, we will investigate the Hyers-Ulam-Rassias stability and the stability by using the alternative fixed point for a generalized cubic functional equation as follows:

$$
\begin{aligned}
& 4 f\left(\sum_{j=1}^{n-1} x_{j}+m x_{n}\right)+4 f\left(\sum_{j=1}^{n-1} x_{j}-m x_{n}\right)+m^{2} \sum_{j=1}^{n-1} f\left(2 x_{j}\right) \\
= & 8 f\left(\sum_{j=1}^{n-1} x_{j}\right)+4 m^{2} \sum_{j=1}^{n-1}\left(f\left(x_{j}+x_{n}\right)+f\left(x_{j}-x_{n}\right)\right)
\end{aligned}
$$

for all $x_{1}, \ldots, x_{n} \in X$, where $m \geq 1$ is an integer number.

\section{Generalized cubic functional equation}

Lemma 2.1. Let $X$ and $Y$ be real vector spaces. A function $f: X \rightarrow Y$ satisfies the functional equation (1.3) if and only if $f$ is cubic. Therefore, every solution of functional equations (1.3) is also a cubic function. 
Proof. Suppose that $f$ satisfies the equation (1.3) for each integer $m \geq 1$. In particular, when $m=2$, we know that $f$ satisfying (1.3) is cubic; see [2, Lemma 2.2]. Hence we note that the case $m=2$ can implies the equation (1.2). It is easy to check that $f(0)=0$ and $f(2 x)=8 f(x)$ for all $x \in X$. First, we start with the case where $m=1$; by letting $x_{1}=x_{1}+x_{n}$ and $x_{1}=x_{1}-x_{n}$, we have

$$
\begin{aligned}
& 4 f\left(\sum_{j=1}^{n-1} x_{j}+2 x_{n}\right)+4 f\left(\sum_{j=1}^{n-1} x_{j}\right)+f\left(2\left(x_{1}+x_{n}\right)\right)+\sum_{j=2}^{n-1} f\left(2 x_{j}\right) \\
= & 8 f\left(\sum_{j=1}^{n-1} x_{j}+x_{n}\right)+4\left(f\left(x_{1}+2 x_{n}\right)+f\left(x_{1}\right)\right) \\
& +4 \sum_{j=2}^{n-1}\left(f\left(x_{j}+x_{n}\right)+f\left(x_{j}-x_{n}\right)\right),
\end{aligned}
$$

and,

$$
\begin{aligned}
& 4 f\left(\sum_{j=1}^{n-1} x_{j}-2 x_{n}\right)+4 f\left(\sum_{j=1}^{n-1} x_{j}\right)+f\left(2\left(x_{1}-x_{n}\right)\right)+\sum_{j=2}^{n-1} f\left(2 x_{j}\right) \\
= & 8 f\left(\sum_{j=1}^{n-1} x_{j}-x_{n}\right)+4\left(f\left(x_{1}-2 x_{n}\right)+f\left(x_{1}\right)\right) \\
& +4 \sum_{j=2}^{n-1}\left(f\left(x_{j}+x_{n}\right)+f\left(x_{j}-x_{n}\right)\right)
\end{aligned}
$$

for all $x_{1}, \ldots, x_{n} \in X$, respectively.

Adding two above equations and using cases where $m=1,2$ and $x_{1}=$ $x, x_{n}=y$, we have

$$
f(x+2 y)+f(x-2 y)+f(2 x)=2 f(x)+4(f(x+y)+f(x-y))
$$

for all $x, y \in X$. Hence $f$ is cubic when $m=1$. Now for any integer $m \geq 3$, letting $x_{1}=x_{1}+x_{n}$ and $x_{1}=x_{1}-x_{n}$ in the equation (1.3) we have

$$
\begin{aligned}
& 4 f\left(\sum_{j=1}^{n-1} x_{j}+(m+1) x_{n}\right)+4 f\left(\sum_{j=1}^{n-1} x_{j}-(m-1) x_{n}\right) \\
& +m^{2} f\left(2\left(x_{1}+x_{n}\right)\right)+m^{2} \sum_{j=2}^{n-1} f\left(2 x_{j}\right) \\
= & 8 f\left(\sum_{j=1}^{n-1} x_{j}+x_{n}\right)+4 m^{2}\left(f\left(x_{1}+2 x_{n}\right)+f\left(x_{1}\right)\right)
\end{aligned}
$$




$$
\begin{aligned}
& +4 m^{2} \sum_{j=2}^{n-1}\left(f\left(x_{j}+x_{n}\right)+f\left(x_{j}-x_{n}\right)\right) \\
& 4 f\left(\sum_{j=1}^{n-1} x_{j}+(m-1) x_{n}\right)+4 f\left(\sum_{j=1}^{n-1} x_{j}-(m+1) x_{n}\right) \\
& +m^{2} f\left(2\left(x_{1}-x_{n}\right)\right)+m^{2} \sum_{j=2}^{n-1} f\left(2 x_{j}\right) \\
& =8 f\left(\sum_{j=1}^{n-1} x_{j}-x_{n}\right)+4 m^{2}\left(f\left(x_{1}-2 x_{n}\right)+f\left(x_{1}\right)\right) \\
& +4 m^{2} \sum_{j=2}^{n-1}\left(f\left(x_{j}+x_{n}\right)+f\left(x_{j}-x_{n}\right)\right)
\end{aligned}
$$

for all $x_{1}, \ldots, x_{n} \in X$, respectively. Adding two above equations and using cases where $m=1,2, m+1$ we have

$$
\begin{aligned}
& 4 f\left(\sum_{j=1}^{n-1} x_{j}+(m-1) x_{n}\right)+4 f\left(\sum_{j=1}^{n-1} x_{j}-(m-1) x_{n}\right)+(m-1)^{2} \sum_{j=1}^{n-1} f\left(2 x_{j}\right) \\
= & 8 f\left(\sum_{j=1}^{n-1} x_{j}\right)+4(m-1)^{2} \sum_{j=1}^{n-1}\left(f\left(x_{j}+x_{n}\right)+f\left(x_{j}-x_{n}\right)\right)
\end{aligned}
$$

for all $x_{1}, \ldots, x_{n} \in X$. By using above method and induction, we infer the cases where $m=1$ or $m=2$, that is, it is cubic. Conversely, suppose that $f$ satisfies the equation (1.2). Because of Lemma 2.2 of [2], we will show that the cases $m=1$ and then $m \geq 3$ integers. Since $f$ satisfies the equation (1.2), we may use the equation (1.3) with $m=2$; see [2, Lemma 2.2]. By putting $x=\sum_{j=1}^{n-1} x_{j}$ and $y=x_{n}$, we have

$$
\begin{aligned}
& f\left(\sum_{j=1}^{n-1} x_{j}+2 x_{n}\right)+f\left(\sum_{j=1}^{n-1} x_{j}-2 x_{n}\right)+f\left(2 \sum_{j=1}^{n-1} x_{j}\right) \\
= & 2 f\left(\sum_{j=1}^{n-1} x_{j}\right)+4 f\left(\sum_{j=1}^{n-1} x_{j}+x_{n}\right)+4 f\left(\sum_{j=1}^{n-1} x_{j}-x_{n}\right) .
\end{aligned}
$$

By using case $m=2$ and $f(2 x)=8 f(x)$, we have the desired result when $m=1$ in the equation (1.3). Next, similar to the previous step and induction with the cases $m=1,2$ and $m=t$, it is easy to show that

$$
4 f\left(\sum_{j=1}^{n-1} x_{j}+(t+1) x_{n}\right)+4 f\left(\sum_{j=1}^{n-1} x_{j}-(t+1) x_{n}\right)+(t+1)^{2} \sum_{j=1}^{n-1} f\left(2 x_{j}\right)
$$




$$
=8 f\left(\sum_{j=1}^{n-1} x_{j}\right)+4(t+1)^{2} \sum_{j=1}^{n-1}\left(f\left(x_{j}+x_{n}\right)+f\left(x_{j}-x_{n}\right)\right)
$$

for all $x_{1}, \ldots, x_{n} \in X$. Thus the $f$ satisfying the equation (1.2) implies the equation (1.3) for each integer $m \geq 1$.

\section{Stability}

Throughout in this section, let $X$ be a normed vector space with norm $\|\cdot\|$ and $Y$ a Banach space with norm $\|\cdot\|$. For the given mapping $f: X \rightarrow Y$, we define

$$
\begin{aligned}
D f\left(x_{1}, \ldots, x_{n}\right):= & 4 f\left(\sum_{j=1}^{n-1} x_{j}+m x_{n}\right)+4 f\left(\sum_{j=1}^{n-1} x_{j}-m x_{n}\right)+m^{2} \sum_{j=1}^{n-1} f\left(2 x_{j}\right) \\
& -8 f\left(\sum_{j=1}^{n-1} x_{j}\right)-4 m^{2} \sum_{j=1}^{n-1}\left(f\left(x_{j}+x_{n}\right)+f\left(x_{j}-x_{n}\right)\right)
\end{aligned}
$$

for all $x_{1}, \ldots, x_{n} \in X$ and each integer $m \geq 1$.

Theorem 3.1. Let $f: X \rightarrow Y$ be a mapping for which there exists a function $\phi: X^{n} \rightarrow[0, \infty)$ such that

$$
\begin{gathered}
\widetilde{\phi}\left(x_{1}, \ldots, x_{n}\right):=\sum_{j=0}^{\infty}\left(\frac{1}{8}\right)^{j} \phi\left(2^{j} x_{1}, \ldots, 2^{j} x_{n}\right)<\infty, \\
\left\|D f\left(x_{1}, \ldots, x_{n}\right)\right\| \leq \phi\left(x_{1}, \ldots, x_{n}\right)
\end{gathered}
$$

for all $x_{1}, \ldots, x_{n} \in X$. Then there exists a unique cubic mapping $C: X \rightarrow Y$ such that

$$
\|f(x)-C(x)\| \leq \frac{1}{8 m^{2} s} \widetilde{\phi}(\underbrace{x, \ldots, x}_{s-\text { terms }}, 0, \ldots, 0)
$$

for all $x \in X$, and each integer $m \geq 1$ and $s \geq 1$.

Proof. By letting $x_{j}=x(j=1, \ldots, s)$ and $x_{k}=0(k=s+1, \ldots, n)$ in the equation (3.3), we have

$$
\left\|f(x)-\frac{1}{8} f(2 x)\right\| \leq \frac{1}{8 m^{2} s} \phi(\underbrace{x, \ldots, x}_{s-\text { terms }}, 0, \ldots, 0)
$$

for all $x \in X$. Replacing $x$ by $2 x$ in the equation (3.5), we have

$$
\left\|f(2 x)-\frac{1}{8} f\left(2^{2} x\right)\right\| \leq \frac{1}{8 m^{2} s} \phi(\underbrace{2 x, \ldots, 2 x}_{s-\text { terms }}, 0, \ldots, 0),
$$


for all $x \in X$. Now, combining equations (3.5) and (3.6), we get

$\left\|f(x)-\left(\frac{1}{8}\right)^{2} f\left(2^{2} x\right)\right\| \leq \frac{1}{8 m^{2} s}\left(\phi(\underbrace{x, \ldots, x}_{s-\text { terms }}, 0, \ldots, 0)+\frac{1}{8} \phi(\underbrace{2 x, \ldots, 2 x}_{s-\text { terms }}, 0, \ldots, 0)\right)$

for all $x \in X$.

Continue this way, we may have

$$
\left\|f(x)-\left(\frac{1}{8}\right)^{t} f\left(2^{t} x\right)\right\| \leq \frac{1}{8 m^{2} s} \sum_{j=0}^{t-1}\left(\frac{1}{8}\right)^{j} \phi(\underbrace{2^{j} x, \ldots, 2^{j} x}_{s-\text { terms }}, 0, \ldots, 0)
$$

for all positive integer $t$ and all $x \in X$. For any positive integer $r$, dividing the equation (3.7) by $8^{r}$ and then substituting $x$ by $2^{r} x$, we have

$$
\begin{aligned}
& \left(\frac{1}{8}\right)^{r}\left\|f\left(2^{r} x\right)-\left(\frac{1}{8}\right)^{t} f\left(2^{r+t} x\right)\right\| \\
\leq & \left(\frac{1}{8}\right)^{r} \cdot \frac{1}{8 m^{2} s} \sum_{j=0}^{t-1}\left(\frac{1}{8}\right)^{j} \phi(\underbrace{2^{r+j} x, \ldots, 2^{r+j} x}_{s-\text { terms }}, 0, \ldots, 0)
\end{aligned}
$$

for all $x \in X$.

By taking $r \rightarrow \infty$, we may conclude that $\left\{\left(\frac{1}{8}\right)^{t} f\left(2^{t} x\right)\right\}$ is a Cauchy sequence in a Banach space $Y$. This implies that the sequence $\left\{\left(\frac{1}{8}\right)^{t} f\left(2^{t} x\right)\right\}$ converges. Hence we can define a function $C: X \rightarrow Y$ by

$$
C(x)=\lim _{t \rightarrow \infty}\left(\frac{1}{8}\right)^{t} f\left(2^{t} x\right)
$$

for all $x \in X$. Then

$$
\begin{aligned}
\left\|D C\left(x_{1}, \ldots, x_{n}\right)\right\| & =\lim _{t \rightarrow \infty}\left(\frac{1}{8}\right)^{t}\left\|\operatorname{Df}\left(2^{t} x, \ldots, 2^{t} x_{n}\right)\right\| \\
& \leq \lim _{t \rightarrow \infty}\left(\frac{1}{8}\right)^{t} \phi\left(2^{t} x_{1}, \ldots, 2^{t} x_{n}\right) \\
& =0
\end{aligned}
$$

for all $x_{1}, \ldots, x_{n} \in X$. That is, $D C\left(x_{1}, \ldots, x_{n}\right)=0$. By Lemma 2.1, the function $C: X \rightarrow Y$ is cubic. It only remains to show that the function $C$ is unique. Let $C^{\prime}: X \rightarrow Y$ be another cubic function satisfying the equation (3.4). Then

$$
\begin{aligned}
\left\|C(x)-C^{\prime}(x)\right\| & =\left(\frac{1}{8}\right)^{t}\left\|C\left(2^{t} x\right)-C^{\prime}\left(2^{t} x\right)\right\| \\
& \leq\left(\frac{1}{8}\right)^{t}\left(\left\|C\left(2^{t} x\right)-f\left(2^{t} x\right)\right\|+\left\|f\left(2^{t} x\right)-C^{\prime}\left(2^{t} x\right)\right\|\right) \\
& \leq\left(\frac{1}{8}\right)^{t} \frac{1}{8} \widetilde{\phi}(\underbrace{2^{t} x, \ldots, 2^{t} x}_{s-\text { terms }}, 0, \ldots, 0)
\end{aligned}
$$

for all $x \in X$. As $t \rightarrow \infty$, we can conclude that $C(x)=C^{\prime}(x)$ for all $x \in X$; that is, $C$ is unique. 
Theorem 3.2. Let $f: X \rightarrow Y$ be a mapping for which there exists a function $\phi: X^{n} \rightarrow[0, \infty)$ such that

$$
\begin{gathered}
\widetilde{\phi}\left(x_{1}, \ldots, x_{n}\right):=\sum_{j=1}^{\infty} 8^{j} \phi\left(2^{-j} x_{1}, \ldots, 2^{-j} x_{n}\right)<\infty, \\
\left\|D f\left(x_{1}, \ldots, x_{n}\right)\right\| \leq \phi\left(x_{1}, \ldots, x_{n}\right)
\end{gathered}
$$

for all $x_{1}, \ldots, x_{n} \in X$. Then there exists a unique cubic mapping $C: X \rightarrow Y$ such that

$$
\|f(x)-C(x)\| \leq \frac{1}{m^{2} s} \widetilde{\phi}(\underbrace{x, \ldots, x}_{s-\text { terms }}, 0, \ldots, 0)
$$

for all $x \in X$, and each integer $m \geq 1$ and $s \geq 1$.

Proof. If $x$ is replaced by $\frac{1}{2} x$ in the equation (3.5) in the proof of Theorem 3.1, we have

$$
|| f(x)-8 f\left(\frac{1}{2} x\right)|| \leq \frac{1}{m^{2}} \phi(\underbrace{\frac{1}{2} x, \ldots, \frac{1}{2} x}_{s-\text { terms }}, 0, \ldots, 0)
$$

for all $x \in X$. The remains of the proof are similar to the proof of Theorem 3.1.

\section{Stability using alternative fixed point}

In this section, we will investigate the stability of the given cubic functional equation (3.1) using the alternative fixed point. Before proceeding the proof, we will state the theorem, the alternative of fixed point.

Theorem 4.1 (The alternative of fixed point [4], [17]). Suppose that we are given a complete generalized metric space $(\Omega, d)$ and a strictly contractive mapping $T: \Omega \rightarrow \Omega$ with Lipschitz constant $L$. Then for each given $x \in \Omega$, either

$$
d\left(T^{n} x, T^{n+1} x\right)=\infty \text { for all } n \geq 0,
$$

or there exists a natural number $n_{0}$ such that

(1) $d\left(T^{n} x, T^{n+1} x\right)<\infty$ for all $n \geq n_{0}$;

(2) The sequence $\left(T^{n} x\right)$ is convergent to a fixed point $y^{*}$ of $T$;

(3) $y^{*}$ is the unique fixed point of $T$ in the set

$$
\triangle=\left\{y \in \Omega \mid d\left(T^{n_{0}} x, y\right)<\infty\right\} ;
$$

(4) $d\left(y, y^{*}\right) \leq \frac{1}{1-L} d(y, T y)$ for all $y \in \triangle$.

Now, let $\phi: X^{n} \rightarrow[0, \infty)$ be a function such that

$$
\lim _{r \rightarrow \infty} \frac{\phi\left(\lambda_{i}^{r} x_{1}, \ldots, \lambda_{i}^{r} x_{n}\right)}{\lambda_{i}^{3 r}}=0
$$

for all $x_{1}, \ldots, x_{n} \in X$, where $\lambda_{i}=2$ if $i=0$ and $\lambda_{i}=\frac{1}{2}$ if $i=1$. 
Theorem 4.2. Suppose that a function $f: X \rightarrow Y$ satisfies the functional inequality

$$
\left\|D f\left(x_{1}, \ldots, x_{n}\right)\right\| \leq \phi\left(x_{1}, \ldots, x_{n}\right)
$$

for all $x_{1}, \ldots, x_{n} \in X$. If there exists $L=L(i)<1$ such that the function

$$
x \mapsto \psi(x)=\frac{1}{m^{2} s} \phi(\underbrace{\frac{1}{2} x, \ldots, \frac{1}{2} x}_{s-\text { terms }}, 0, \ldots, 0)
$$

has the property

$$
\psi(x) \leq L \cdot \lambda_{i}^{3} \cdot \psi\left(\frac{x}{\lambda_{i}}\right)
$$

for all $x \in X$, then there exists a unique cubic function $C: X \rightarrow Y$ such that the inequality

$$
\|f(x)-C(x)\| \leq \frac{L^{1-i}}{1-L} \psi(x)
$$

holds for all $x \in X$.

Proof. Consider the set

$$
\Omega=\{g \mid g: X \rightarrow Y\}
$$

and introduce the generalized metric on $\Omega$,

$$
d(g, h)=d_{\psi}(g, h)=\inf \{K \in(0, \infty) \mid\|g(x)-h(x)\| \leq K \psi(x), x \in X\} .
$$

It is easy to show that $(\Omega, d)$ is complete. Now we define a function $T: \Omega \rightarrow \Omega$ by

$$
\operatorname{Tg}(x)=\frac{1}{\lambda_{i}^{3}} g\left(\lambda_{i} x\right)
$$

for all $x \in X$. Note that for all $g, h \in \Omega$,

$$
\begin{aligned}
d(g, h)<K & \Rightarrow\|g(x)-h(x)\| \leq K \psi(x) \text { for all } x \in X, \\
& \Rightarrow\left\|\frac{1}{\lambda_{i}^{3}} g\left(\lambda_{i} x\right)-\frac{1}{\lambda_{i}^{3}} h\left(\lambda_{i} x\right)\right\| \leq \frac{1}{\lambda_{i}^{3}} K \psi\left(\lambda_{i} x\right) \text { for all } x \in X, \\
& \Rightarrow\left\|\frac{1}{\lambda_{i}^{3}} g\left(\lambda_{i} x\right)-\frac{1}{\lambda_{i}^{3}} h\left(\lambda_{i} x\right)\right\| \leq L K \psi(x) \text { for all } x \in X, \\
& \Rightarrow d(T g, T h) \leq L K .
\end{aligned}
$$

Hence we have that

$$
d(T g, T h) \leq L d(g, h)
$$

for all $g, h \in \Omega$, that is, $T$ is a strictly self-mapping of $\Omega$ with the Lipschitz constant $L$. By setting $y=0$, we have the equation (3.5) as in the proof of Theorem 3.1 and we use the equation (4.3) with the case where $i=0$, which is reduced to

$$
\left\|f(x)-\frac{1}{8} f(2 x)\right\| \leq \frac{1}{m^{2} s} \frac{1}{2^{3}} \psi(2 x) \leq L \psi(x)
$$


for all $x \in X$, that is, $d(f, T f) \leq L=L^{1}<\infty$. Now, replacing $x$ by $\frac{1}{2} x$ in the equation (3.5), multiplying 8 , and using the equation (4.3) with the case where $i=1$, we have that

$$
\left\|f(x)-2^{3} f\left(\frac{x}{2}\right)\right\| \leq \psi(x)
$$

for all $x \in X$, that is, $d(f, T f) \leq 1=L^{0}<\infty$. In both cases we can apply the fixed point alternative and since $\lim _{r \rightarrow \infty} d\left(T^{r} f, C\right)=0$, there exists a fixed point $C$ of $T$ in $\Omega$ such that

$$
C(x)=\lim _{r \rightarrow \infty} \frac{f\left(\lambda_{i}^{r} x\right)}{\lambda_{i}^{3 r}}
$$

for all $x \in X$. Letting $x_{j}=\lambda_{i}^{r} x_{j}(j=1, \ldots, n)$ in the equation (4.1) and dividing by $\lambda_{i}^{3 r}$,

$$
\begin{aligned}
\left\|D C\left(x_{1}, \ldots, x_{n}\right)\right\| & =\lim _{r \rightarrow \infty} \frac{\left\|D f\left(\lambda_{i}^{r} x_{1}, \ldots, \lambda_{i}^{r} x_{n}\right)\right\|}{\lambda^{3 r}} \\
& \leq \lim _{r \rightarrow \infty} \frac{\left\|\phi\left(\lambda_{i}^{r} x_{1}, \ldots, \lambda_{i}^{r} x_{n}\right)\right\|}{\lambda^{3 r}}=0
\end{aligned}
$$

for all $x_{1}, \ldots, x_{n} \in X$; that is, it satisfies the equation (1.3). By Lemma 2.1, the $C$ is cubic. Also, the fixed point alternative guarantees that such a $C$ is the unique function such that

$$
\|f(x)-C(x)\| \leq K \psi(x)
$$

for all $x \in X$ and some $K>0$. Again using the fixed point alternative, we have

$$
d(f, C) \leq \frac{1}{1-L} d(f, T f) .
$$

Hence we may conclude that

$$
d(f, C) \leq \frac{L^{1-i}}{1-L}
$$

which implies the equation (4.4).

\section{References}

[1] P. W. Cholewa, Remarks on the stability of functional equations, Aequationes Math. 27 (1984), no. 1-2, 76-86.

[2] H. Y. Chu and D. S. Kang, On the stability of an $n$-dimensional cubic functional equation, J. Math. Anal. Appl. 325 (2007), no. 1, 595-607.

[3] S. Czerwik, On the stability of the quadratic mapping in normed spaces, Abh. Math. Sem. Univ. Hamburg 62 (1992), 59-64.

[4] J. B. Diaz and B. Margolis, A fixed point theorem of the alternative for contractions on a generalized complete metric space, Bull. Amer. Math. Soc. 74 (1968), 305-309.

[5] Z. Gajda, On stability of additive mappings, Internat. J. Math. Math. Sci. 14 (1991), no. 3, 431-434.

[6] P. Găvruţa, A generalization of the Hyers-Ulam-Rassias stability of approximately additive mappings, J. Math. Anal. Appl. 184 (1994), no. 3, 431-436.

[7] D. H. Hyers, On the stability of the linear functional equation, Proc. Nat. Acad. Sci. U. S. A. 27 (1941), 222-224. 
[8] D. H. Hyers, G. Isac, and Th. M. Rassias, Topics in Nonlinear Analysis and Applications, World Scientific Publishing Company, Singapore, New jersey, London, 1997.

[9] D. H. Hyers and Th. M. Rassias, Approximate homomorphisms, Aequationes Math. 44 (1992), no. 2-3, 125-153.

[10] G. Isac and Th. M. Rassias, Stability of $\Psi$-additive mappings: applications to nonlinear analysis, Internat. J. Math. Math. Sci. 19 (1996), no. 2, 219-228.

[11] K.-W. Jun and H.-M. Kim, The generalized Hyers-Ulam-Rassias stability of a cubic functional equation, J. Math. Anal. Appl. 274 (2002), no. 2, 867-878.

[12] Th. M. Rassias, On the stability of the linear mapping in Banach spaces, Proc. Amer. Math. Soc. 72 (1978), no. 2, 297-300.

[13] 251 (2000), no. 1, 264-284.

[14] On the stability of functional equations and a problem of Ulam, Acta Appl. Math. 62 (2000), no. 1, 23-130.

[15] _ The problem of S. M. Ulam for approximately multiplicative mappings, J. Math. Anal. Appl. 246 (2000), no. 2, 352-378.

[16] _ Problem 16; 2, Report of the 27th International Symp. on Functional Equations, Aequationes Math. 39 (1990), 292-293; 309.

[17] I. A. Rus, Principles and Appications of Fixed Point Theory, Ed. Dacia, Cluj-Napoca, 1979.

[18] F. Skof, Local properties and approximation of operators, Rend. Sem. Mat. Fis. Milano 53 (1983), 113-129.

[19] S. M. Ulam, Problems in Morden Mathematics, Wiley, New York, 1960.

HEEJEONG KOH

Department of Mathematics Education

College of Education

DANKOOK UNIVERSITY

GYEONGI 448-701, KOREA

E-mail address: khjmath@dankook.ac.kr

DongSeung Kang

Department of Mathematics Education

College of Education

DANKOOK UNIVERSITY

GYEONGI 448-701, KoREA

E-mail address: dskang@dankook.ac.kr 